\title{
Cornu Cervi Pantotrichum Supplementation Improves Exercise Performance and Protects against Physical Fatigue in Mice
}

\author{
Chi-Chang Huang ${ }^{1}$, Yi-Ming Chen ${ }^{1, \dagger}$, Nai-Wen Kan ${ }^{2,3, \dagger}{ }^{,}$, Hui-Ling Chao ${ }^{1}$, Chin-Shan Ho ${ }^{1}$ and \\ Mei-Chich Hsu ${ }^{4, *}$
}

1 Graduate Institute of Sports Science, College of Exercise and Health Sciences, National Taiwan Sport University, Taoyuan 33301, Taiwan

2 Graduate Institute of Athletics and Coaching Science, College of Sports and Athletics, National Taiwan Sport University, Taoyuan 33301, Taiwan

3 Center for Liberal Arts, Taipei Medical University, Taipei 11031, Taiwan

4 Department of Sports Medicine, Kaohsiung Medical University, Kaohsiung 80708, Taiwan

$\dagger$ These authors contributed equally to this work.

* Author to whom correspondence should be addressed; E-Mail: meichich@kmu.edu.tw or meichich@gmail.com; Tel.: +886-7-312-1101 (ext. 2793-613).

Received: 24 February 2014; in revised form: 11 April 2014 / Accepted: 11 April 2014 /

Published: 15 April 2014

\begin{abstract}
Cornu cervi pantotrichum (CCP) is a well-known yang-invigorating agent used in traditional Chinese medicine that can nourish the blood, tonify qi, and invigorate bones and tendons with multifunctional bioactivities. However, evidence on the effects of CCP on exercise performance and physical fatigue is limited. We evaluated the potential beneficial effects of ethanolic extract from CCP on ergogenic and antifatigue functions following a physiological challenge. Male ICR mice from four groups ( $n=8$ per group) were orally administered CCP for 14 days at 0,2054 , and $4108 \mathrm{mg} / \mathrm{kg} / \mathrm{day}$, and were respectively designated as the vehicle, CCP-1X, and CCP-2X groups. The physical performance and antifatigue function were evaluated using forelimb grip strength and exhaustive swimming time as well as serum levels of lactate, ammonia, glucose, and creatine kinase after a 15 -min swimming exercise. The results indicated that CCP-1X supplementation significantly improved grip strength; reduced fatigue-associated biochemical indices, including lactate and ammonia levels; and ameliorated skeletal muscle injury induced by acute exercise challenge. A trend analysis revealed that CCP supplementation significantly increased grip strength and dose-dependently reduced serum
\end{abstract}


alkaline phosphatase, uric acid, triacylglycerol, and glucose levels in healthy mice.

Therefore, CCP is a potential agent with an antifatigue pharmacological effect.

Keywords: deer antler; grip strength; antifatigue

\section{Introduction}

Cornu cervi pantotrichum (CCP), or deer antler, is a well-known traditional Chinese medicine (TCM) recorded in the Compendium of Materia Medica (Bencao Gangmu) by Li Shi-Zhen approximately 500 years ago. CCP is believed to invigorate the spleen, strengthen bone and skeletal muscles, and promote blood flow [1]. The main nutrients and chemical constituents in CCP are proteins, amino acids, minerals, lipids, polypeptides, polysaccharides, phospholipids, and trace elements including $\mathrm{Ca}, \mathrm{P}, \mathrm{K}, \mathrm{Al}, \mathrm{Zn}, \mathrm{Cu}$, and $\mathrm{Fe}[1,2]$. CCP has been extensively used in TCM as an antioxidant and for improving sexual function, postponing fatigue, and treating various disorders such as osteoporosis [1,3,4]. In addition, both in vitro and in vivo pharmacological studies have demonstrated that CCP exhibits antithrombotic [5], endocrine anticoagulation and antithrombotic [6,7], antiaging [8], immunomodulatory, anticancer, and antiinflammatory properties [1]. However, relatively few studies have directly addressed the potential ergogenic or antifatigue functions of CCP.

Fatigue is defined as physical and/or mental weariness resulting in negative effects on exercise intensity, work performance, family life, and social relationships [9]. Physical fatigue can be accompanied by deterioration in functional performance [10]. At least two mechanisms can explain the occurrence of physical fatigue: oxidative stress and energy exhaustion [11]. Exhaustive or intensive exercise can lead to the accumulation of excess reactive free radicals, resulting in tissue damage. Exhaustion theory suggests that energy source depletion and excess metabolite accumulation can lead to fatigue $[12,13]$. In this study, we evaluated the potential ergogenic and antifatigue effects of CCP by using our previously established in vivo platform [14,15].

\section{Results and Discussion}

\subsection{Body Weight and Other Metabolism-Related Organ Weights}

Morphological data from each experimental group are summarized in Table 1. The initial and final body weights, food intake, and water consumption did not differ among the three treatment groups. In addition, the groups did not differ in absolute weights of the liver, muscle, heart, lung, kidney, testis, epididymal fat pad (EFP), and brown adipose tissue (BAT).

Table 1. General characteristics of the experimental groups.

\begin{tabular}{ccccc}
\hline Characteristic & Vehicle & CCP-1X & CCP-2X & Trend analysis \\
\hline Initial BW (g) & $30.1 \pm 0.3$ & $29.4 \pm 0.4$ & $30.5 \pm 0.3$ & 0.5798 \\
Final BW (g) & $33.8 \pm 0.8$ & $34.6 \pm 0.6$ & $34.7 \pm 0.7$ & 0.5583 \\
Food intake (g/day) & $6.91 \pm 0.28$ & $7.23 \pm 0.22$ & $7.29 \pm 0.24$ & 0.3765 \\
\hline
\end{tabular}


Table 1. Cont.

\begin{tabular}{ccccc}
\hline Characteristic & Vehicle & CCP-1X & CCP-2X & Trend analysis \\
\hline Water intake (mL/day) & $7.93 \pm 0.14$ & $7.51 \pm 0.22$ & $8.27 \pm 0.25$ & 0.4492 \\
Liver (g) & $1.86 \pm 0.06$ & $1.87 \pm 0.02$ & $1.81 \pm 0.04$ & 0.2206 \\
Muscle (g) & $0.34 \pm 0.01$ & $0.32 \pm 0.01$ & $0.35 \pm 0.01$ & 0.7708 \\
Heart (g) & $0.21 \pm 0.00$ & $0.21 \pm 0.01$ & $0.22 \pm 0.00$ & 0.5426 \\
Lung (g) & $0.32 \pm 0.04$ & $0.32 \pm 0.04$ & $0.35 \pm 0.04$ & 0.4720 \\
Kidney (g) & $0.57 \pm 0.02$ & $0.55 \pm 0.01$ & $0.58 \pm 0.02$ & 0.8445 \\
Testis (g) & $0.21 \pm 0.01$ & $0.23 \pm 0.01$ & $0.21 \pm 0.00$ & 0.2130 \\
EFP (g) & $0.65 \pm 0.04$ & $0.56 \pm 0.05$ & $0.58 \pm 0.04$ & 0.4589 \\
BAT (g) & $0.12 \pm 0.01$ & $0.12 \pm 0.01$ & $0.12 \pm 0.01$ & 0.0882 \\
\hline
\end{tabular}

Data are mean \pm SEM for $n=8$ mice in each group. Muscle mass includes both gastrocnemius and soleus muscles in the back part of the lower legs. EFP: epididymal fat pad; BAT, brown adipose tissue.

\subsection{Effect of CCP Supplementation on Forelimb Grip Strength and Exercise Performance in a} Weight-Loaded Swimming Test

As shown in Figure 1, the forelimb grip strength values in the vehicle, CCP-1X, and CCP-2X groups were 121, 137, and $143 \mathrm{~g}$, respectively; the values of the CCP-1X and CCP-2X groups were significantly 1.13- $(p=0.0103)$ and 1.19-fold $(p=0.0008)$ higher than those of the vehicle group. In the trend analysis, absolute forelimb grip strength dose-dependently increased as the CCP dose $(p<0.0001)$ increased. A regulatory training program is required to increase grip strength [16]; however, the results indicated that CCP supplementation benefited grip strength even though the mice did not undergo a training intervention. Thus, short-term CCP treatment can benefit grip strength when no training intervention is implemented. Previous reports have shown that 7 to 21 days of supplementation with plant extracts or resveratrol or long-term supplementation with agents such as whey protein improves the grip strength of untrained and undertrained animals [16]. Thus, CCP, a substance of animal origin used in TCM, may be an alternative supplement for promoting body strength in a programmed training protocol.

Figure 1. Effect of CCP supplementation on forelimb grip strength performance. Data are presented as the mean \pm SEM of 8 mice in each group. Different letters $(a, b)$ indicate a significant difference at $p<0.05$ by one-way ANOVA.

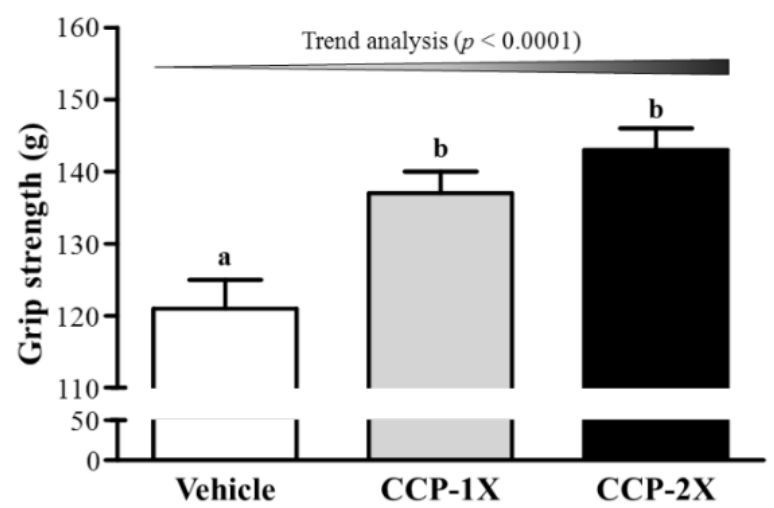


Exercise endurance is another variable used to evaluate exercise endurance performance. In our study, the exercise endurance levels of mice subjected to a swimming test and administered the vehicle, CCP-1X, and CCP-2X were $7.1 \pm 0.7,14.7 \pm 6.2$, and $6.8 \pm 1.3 \mathrm{~min}$, respectively. However, no significant differences in swimming times were observed among the vehicle and CCP treatment groups. One recent review reported that aqueous extract of deer antler base prolonged the endurance exercise time in untrained test animals [1]. However, a previous report showed that 10 weeks of elk velvet antler supplementation combined with training did not improve the rowing performance of male and female rowers [17]. Based on these results, we suggest that CCP, ethanolic extract of deer antler, improves body strength but not endurance performance in the absence of training. Therefore, further investigation is required to elucidate the effects of long-term CCP supplementation combined with exercise training on endurance performance.

\subsection{Effect of CCP Supplementation on Serum Lactate, Ammonia, Glucose, and Creatine Kinase Levels after Acute Exercise Challenge}

Muscle fatigue after exercise can be evaluated using crucial biochemical indicators including lactate, ammonia, glucose, and creatine kinase (CK) after exercise [18]. During high-intensity exercise, muscles must obtain sufficient energy through anaerobic glycolysis, and a substantial amount of lactate is produced through glycolysis metabolism. The increased lactate level reduces the $\mathrm{pH}$ value, resulting in various biochemical and physiological side effects on glycolysis, phosphofructokinase, and muscular contractions caused by calcium ion release [19]. In the present study, respective lactate levels in the vehicle, CCP-1X, and CCP-2X groups were $8.7 \pm 1.1,6.3 \pm 0.9$, and $8.2 \pm 0.9 \mathrm{mmol} / \mathrm{L}$; only the lactate levels of the mice that received CCP-1X supplementation were significantly lower than those of mice that received the vehicle treatment (27.94\% difference; $p<0.0001$; Figure $2 \mathrm{a})$.

Figure 2. Effect of CCP supplementation on serum levels of lactate (a), ammonia (b), glucose (c), and CK (d) after a 15-min swim test without weight-loading. Data represent the mean \pm SEM of 8 mice in each group. Columns with different letters $(a, b)$ significantly difference, $p<0.05$ by a one-way ANOVA.

(a)

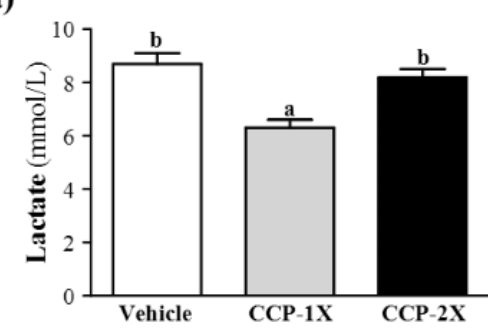

(c)

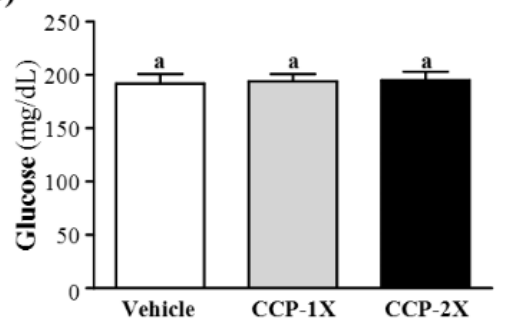

(b)

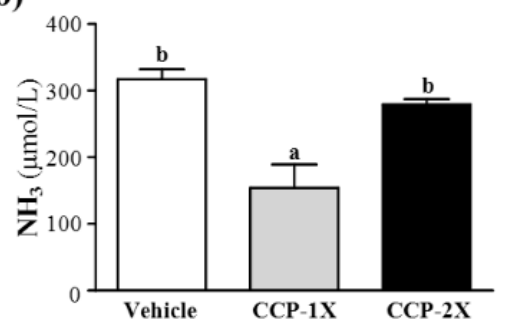

(d)

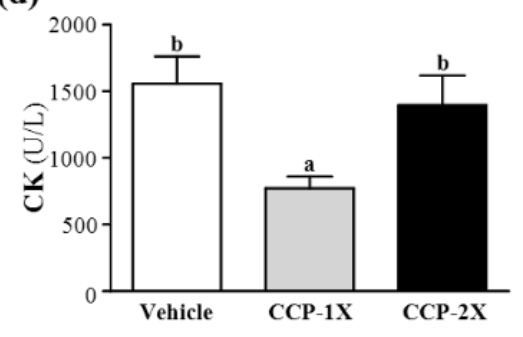


Ammonia, a metabolite of proteins and amino acids, was linked to fatigue as early as 1922 . The immediate source of ammonia produced from the deamination of AMP to inosine monophosphate through the purine nucleotide cycle is the greatest during intensive exercise when the rate of ATP use may exceed the rate of ATP production. Muscle fatigue is associated with the deamination of adenine nucleotides, and increased deamination of AMP coincides with decreases in phosphocreatine and $\mathrm{pH}$ values as well as failure of the contraction process. Peripheral and central fatigue levels are related to increased ammonia levels during exercise [20]. The serum ammonia levels in the vehicle, CCP-1X, and CCP-2X groups were $317 \pm 47,154 \pm 112$, and $279 \pm 26 \mu \mathrm{mol} / \mathrm{L}$, respectively; only the serum ammonia levels of mice that received CCP-1X supplementation were significantly lower than those of mice that received the vehicle treatment ( $51.26 \%$ difference; $p=0.0002$; Figure $2 b$ ).

Glucose, a breakdown product of tissue glycogen, is released as a circulating substrate and used as energy after intense exercise [21]. During exercise, the plasma glucose level increases through the combined action of glucagon, epinephrine, norepinephrine, and cortisol. Although insulin enables glucose to enter a body cell, glucose declines during prolonged exercise. Exercise and muscle contractions increase glucose uptake in skeletal muscles through a mechanism that is independent of the insulin signaling pathway [22]. Figure $2 \mathrm{c}$ shows that the respective levels of serum glucose in the vehicle, CCP-1X, and CCP-2X groups were $192 \pm 28,194 \pm 23$, and $195 \pm 24 \mathrm{mg} / \mathrm{dL}$. No significant differences were observed in serum glucose levels among the vehicle and CCP treatment groups after a 15-min swim test.

Serum CK is a crucial clinical biomarker for various types of muscle damage, such as muscular dystrophy, severe muscle breakdown, myocardial infarction, autoimmune myositides, and acute renal failure. CK activity levels in the vehicle, CCP-1X, and CCP-2X groups were $1558 \pm 643,772 \pm 277$, and $1396 \pm 704 \mathrm{U} / \mathrm{L}$, respectively, and only CK levels in the CCP-1X group were significantly lower than those in the vehicle group (50.47\% difference; $p=0.0121$; Figure $2 \mathrm{~d}$ ).

Thus, our results suggested that a daily recommended dose of $\mathrm{CCP}$ efficiently relieved fatigueassociated biochemical indices, including lactate and ammonia levels, and ameliorated skeletal muscle injury induced by acute exercise challenge.

\subsection{Effect of CCP Supplementation on Biochemical Analyses at the End of the Experiment}

In the present study, we observed the beneficial effects of a daily recommended dose of CCP supplementation on grip strength performance and fatigue-associated biochemical indices postexercise. Therefore, we examined whether administering CCP treatments for 14 days exerts negative effects on other biochemical markers in healthy mice. In a previous acute study, rats of both sexes were exposed to a dose of $2,000 \mathrm{mg} / \mathrm{kg}$ body weight. No mortality or other signs of toxicity were observed during 14 days of observation [23]. Furthermore, our histopathological examinations revealed that CCP supplementation for 14 days yielded no adverse effects in major organs such as the liver, skeletal muscle, heart, kidney, lung, and testis (Figure 3). We determined that the liver- and kidney-related biochemical parameters were nontoxic in CCP-treated mice (Table 2). Therefore, the dose of CCP supplementation used in this study was safe. Table 2 shows that the serum ALP, TC, TG, and glucose levels in the CCP-2X group were significantly $25.19 \%(p=0.0094), 10.03 \%(p=0.0465), 47.73 \%$ $(p<0.0001)$, and $12.39 \%(p=0.0100)$, respectively, lower than those of the vehicle group. The trend 
analysis revealed that CCP supplementation dose-dependently reduced serum levels of ALP $(p<0.0001)$, UA $(p=0.0111)$, TG $(p<0.0001)$, and glucose $(p=0.0055)$. Our results were consistent with those of a previous report indicating that velvet antler supplementation significantly reduced serum ALP levels compared with those of a control group [4]. CCP supplementation produces no side effects in vivo and exhibits potential for application in measuring blood glucose, UA, and hyperlipidemia according to our in vivo data.

Table 2. Biochemical analysis of the CCP treatment groups at the end of the experiment.

\begin{tabular}{ccccc}
\hline Parameter & Vehicle & CCP-1X & CCP-2X & Trend analysis \\
\hline AST (U/L) & $78 \pm 6$ & $78 \pm 10$ & $72 \pm 6$ & 0.4773 \\
ALT (U/L) & $53 \pm 4$ & $44 \pm 4^{\mathrm{a}}$ & $46 \pm 2$ & 0.1381 \\
ALP (U/L) & $444 \pm 29^{\mathrm{b}}$ & $382 \pm 35^{\text {ab }}$ & $332 \pm 15^{\mathrm{a}}$ & $<0.0001(\downarrow)$ \\
LDH (U/L) & $396 \pm 26$ & $451 \pm 37$ & $428 \pm 21$ & 0.3274 \\
CK (U/L) & $245 \pm 33$ & $282 \pm 90$ & $187 \pm 25$ & 0.3077 \\
Albumin (g/dL) & $3.3 \pm 0.1$ & $3.4 \pm 0.0$ & $3.3 \pm 0.1$ & 1.0000 \\
TP (g/dL) & $5.6 \pm 0.1$ & $5.8 \pm 0.1$ & $5.6 \pm 0.1$ & 0.9163 \\
BUN (mg/dL) & $25.2 \pm 0.9$ & $25.2 \pm 1.1$ & $22.4 \pm 0.9$ & 0.0840 \\
Creatinine (mg/dL) & $0.30 \pm 0.0$ & $0.31 \pm 0.02$ & $0.33 \pm 0.01$ & 0.0600 \\
UA (mg/dL) & $1.16 \pm 0.12$ & $1.06 \pm 0.13$ & $0.89 \pm 0.05$ & $0.0111(\downarrow)$ \\
TC (mg/dL) & $160 \pm 4^{\mathrm{b}}$ & $168 \pm 5^{\mathrm{b}}$ & $144 \pm 7^{\mathrm{a}}$ & 0.0884 \\
TG (mg/dL) & $162 \pm 13^{\mathrm{b}}$ & $137 \pm 10^{\mathrm{b}}$ & $84 \pm 8^{\mathrm{a}}$ & $<0.0001(\downarrow)$ \\
Glucose (mg/dL) & $201 \pm 5^{\mathrm{b}}$ & $186 \pm 6^{\text {ab }}$ & $176 \pm 7^{\mathrm{a}}$ & $0.0055(\downarrow)$ \\
\hline
\end{tabular}

Values are mean \pm SEM for $\mathrm{n}=8$ mice per group. Values in the same row with different superscript letters $(\mathrm{a}, \mathrm{b})$ differ significantly, $p<0.05$ by one-way ANOVA. AST, aspartate aminotransferase (AST); alanine aminotransferase (ALT); alkaline phosphatase (ALP); creatine kinase (CK); lactate dehydrogenase (LDH); total protein (TP); blood urea nitrogen (BUN); uric acid (UA); total cholesterol (TC); triacylglycerol (TG).

\subsection{Effect of CCP Supplementation on Histological Examinations at the End of the Experiment}

Figure 3 shows that the three groups did not differ according to histological observations of the liver, muscle, heart, kidney, lung, and testis.

Figure 3. Effect of CCP supplementation on the morphology of liver (A), skeletal muscle (B), heart (C), kidney (D), lung (E), and testis (F). Specimens were photographed with a light microscope (Olympus BX51). (H\&E stain, magnification: $\times 200$, Scale bar, $40 \mu \mathrm{m}$ ).

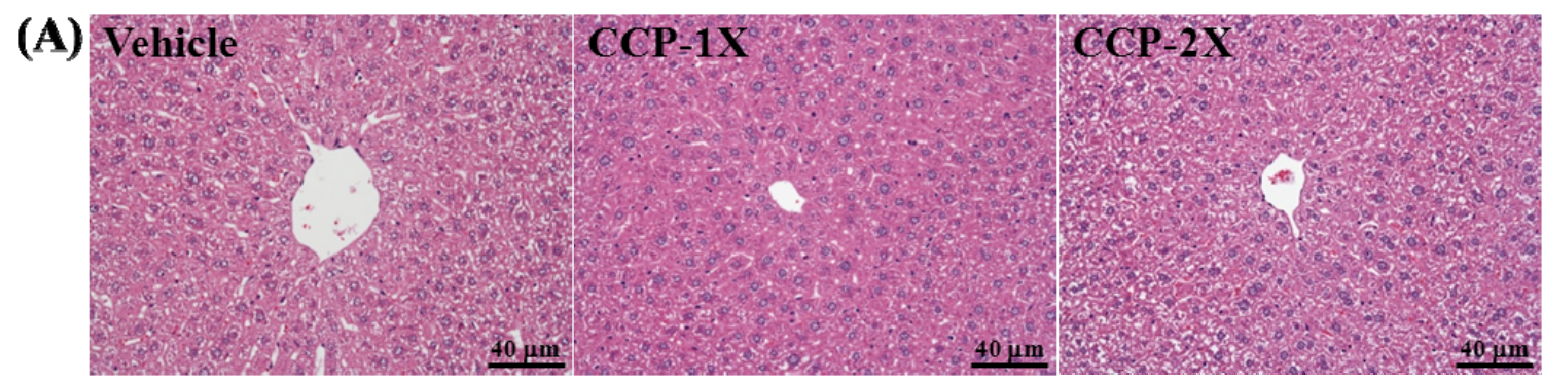


Figure 3. Cont.
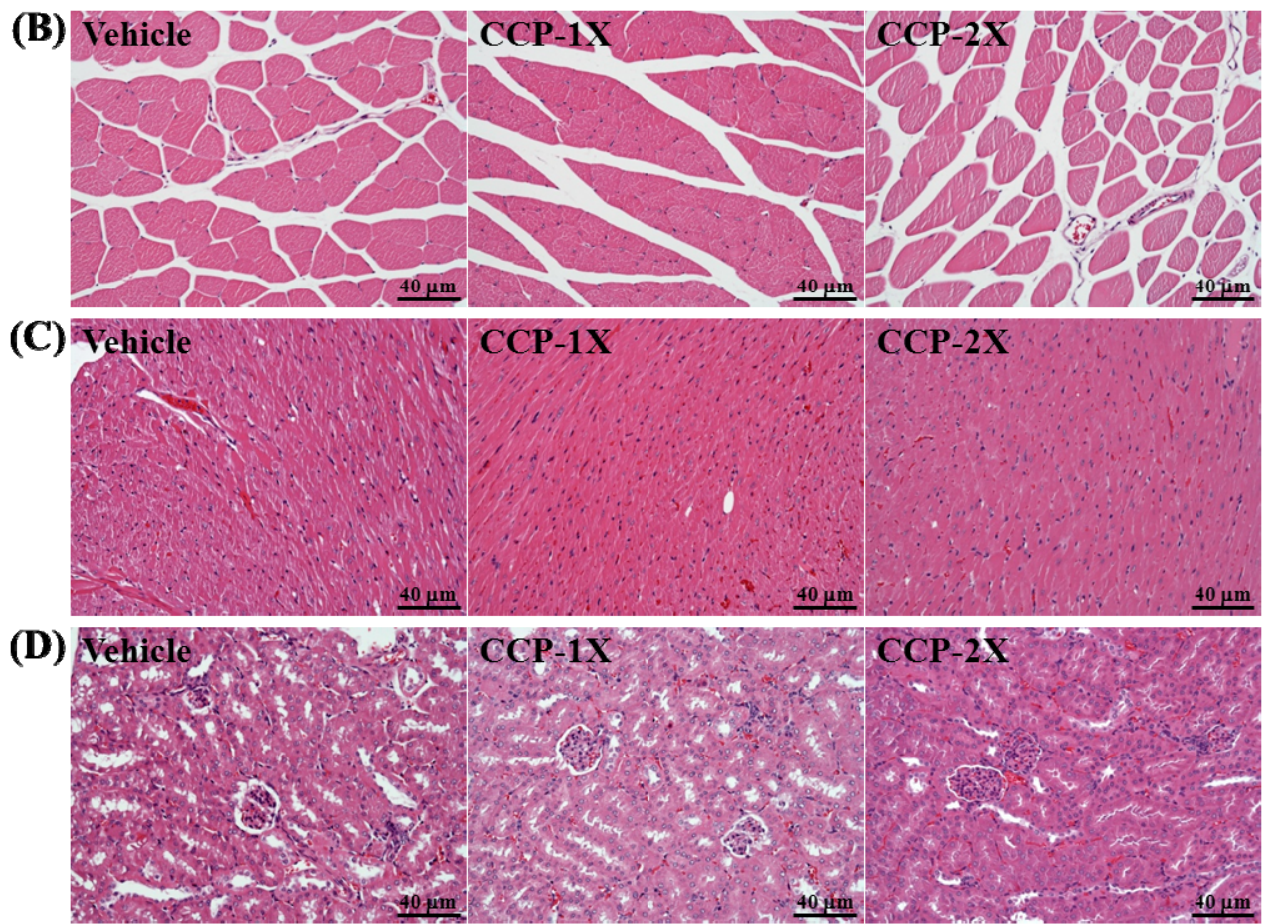

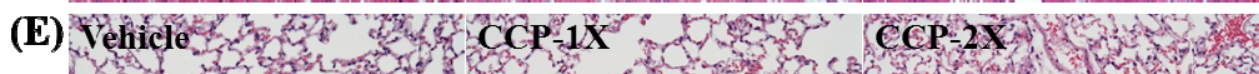

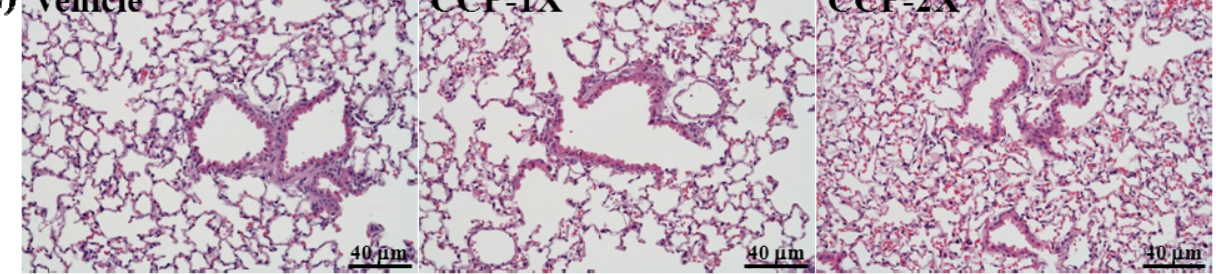

(F) Nehicle:

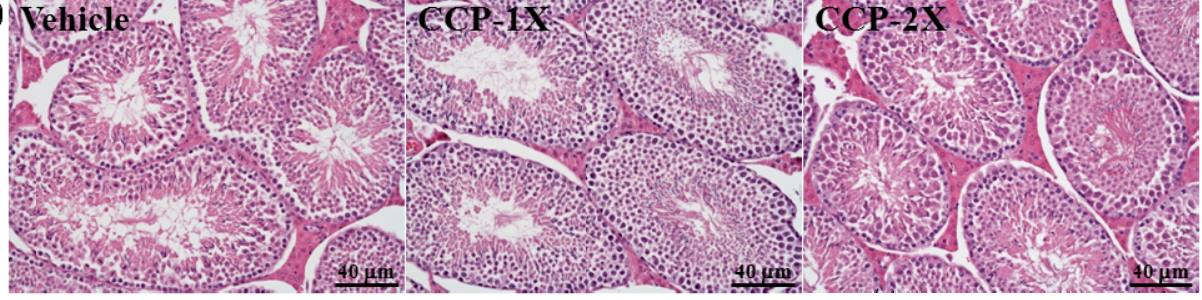

\section{Experimental}

\subsection{Materials, Animals, and Experiment Design}

CCP ethanolic extract powder was purchased from International Total Solution (Taiwan), Inc. (Taipei City, Taiwan). Figure 4 shows the protocol used for preparing CCP and the experimental design of the study. Male ICR-strain mice (6 weeks old) with a specific pathogen-free condition were purchased from BioLASCO (Yi-Lan, Taiwan). All animals were provided with a standard laboratory diet (No. 5001; PMI Nutrition International, Brentwood, MO, USA) and distilled water ad libitum, and housed under a 12-h light/12-h dark cycle at room temperature $\left(22 \pm 1{ }^{\circ} \mathrm{C}\right)$ and $50 \%-60 \%$ humidity. The Institutional Animal Care and Use Committee (IACUC) of National Taiwan Sport University evaluated all animal experiments, and this study conformed to the guidelines of protocol IACUC10109 and was approved by the IACUC ethics committee. 
Figure 4. Protocol for preparation of ethanolic extract from Cornu cervi pantotrichum and experimental design of the study.

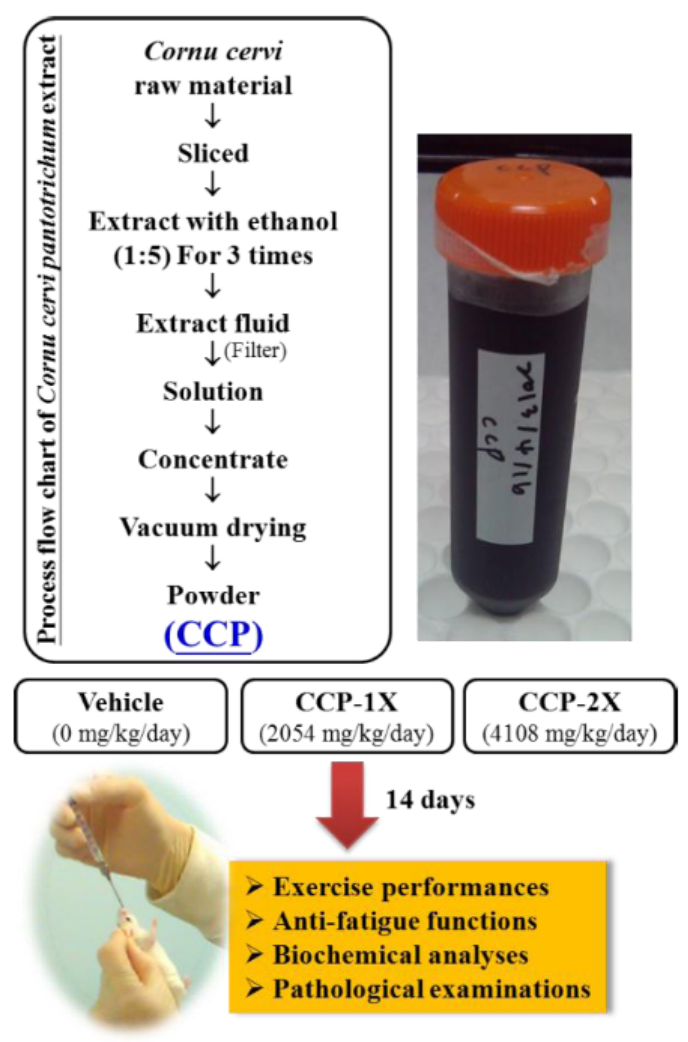

The recommended dose of CCP for humans is approximately $10 \mathrm{~g}$ with a normal diet. The CCP dose $(2054 \mathrm{mg} / \mathrm{kg})$ administered to mice in this study was converted from a human-equivalent dose (HED) based on body surface area by using the following formula provided by the U.S. Food and Drug Administration: Assuming a human weight of $60 \mathrm{~kg}$, the HED for $10(\mathrm{~g}) \div 60(\mathrm{~kg})=0.167 \times 12.3=\mathrm{a}$ mouse dose of $2054 \mathrm{mg} / \mathrm{kg}$. The conversion coefficient 12.3 was used to account for differences in body surface area between a mouse and a human, as described in our recent study [16].

After a 1-week adaptation period, all mice were randomly assigned to three groups ( $n=8$ per group) for CCP treatment: (1) the vehicle treatment; (2) 2,054 mg/kg of CCP (CCP-1X), or (3) $4108 \mathrm{mg} / \mathrm{kg}$ of CCP (CCP-2X). The vehicle group received the same volume of solution equivalent to the individual body weight. Both the vehicle and CCP were administered orally to each animal for 14 days.

\subsection{Forelimb Grip Strength}

A low-force testing system (Model-RX-5, Aikoh Engineering, Nagoya, Japan) was used to measure the forelimb grip strength of mice undergoing vehicle or CCP treatments. The amount of tensile force exerted by each mouse was measured using a force transducer equipped with a metal bar ( $2 \mathrm{~mm}$ in diameter and $7.5 \mathrm{~cm}$ in length). The detailed procedures have been described in our previous reports $[14,15]$. The test of forelimb grip strength was performed after the vehicle or CCP was administered consecutively for 14 days and $1 \mathrm{~h}$ after the last treatment. The maximal force (in grams) recorded using this low-force system was used as the grip strength. 


\subsection{Swimming Exercise Performance Test}

The swim-to-exhaustion exercise test involved applying constant loads corresponding to $5 \%$ of the body weight of each mouse to evaluate endurance, as described in our previous report [24]. The endurance of each mouse was recorded as the time from the beginning of the test to exhaustion, which was determined by observing loss of coordinated movements and failure to return to the surface within $7 \mathrm{~s}$.

\subsection{Determination of Fatigue-Associated Biochemical Indices}

The effects of CCP supplementation on fatigue-associated biochemical variables were evaluated postexercise according to the procedure described in our previous reports [14,15,24]. At $1 \mathrm{~h}$ after CCP supplementation, all animals underwent a 15-min swim test without weight loading, after which a blood sample was immediately collected and centrifuged at $1,500 \times g$ and $4{ }^{\circ} \mathrm{C}$ for 10 min to prepare the serum sample. Serum lactate, ammonia, glucose, and CK levels were quantified using an autoanalyzer (Hitachi 7060, Hitachi, Tokyo, Japan).

\subsection{Blood Biochemical Assessments and Histological Staining of Tissues}

At the end of the experimental period, all mice were finally sacrificed by asphyxiating them with 95\% $\mathrm{CO}_{2}$, and blood was immediately collected at rest. Serum was collected by conducting centrifugation, and the clinical biochemical variables were measured using an autoanalyzer, as shown in Table 2 (Hitachi 7060). Various tissues were collected and fixed in 10\% formalin after the mice were sacrificed. The tissues were cut transversely or longitudinally to obtain ventricular sections or 4-chamber cross-sections, respectively. The tissues were subsequently embedded in paraffin and cut into $4-\mu \mathrm{m}$ thick slices for morphological and pathological evaluations. The tissue sections were stained with hematoxylin and eosin (H\&E) and examined using a light microscope equipped with a CCD camera (BX-51, Olympus, Tokyo, Japan) by a veterinary pathologist.

\subsection{Statistical Analysis}

All data are expressed as the mean \pm SEM. Statistical differences among groups were analyzed by conducting a one-way analysis of variance (ANOVA), and the Cochran-Armitage test was used to perform a dose-effect trend analysis by employing SAS Version 9.0 (SAS Institute, Cary, NC, USA). A $p$ value $<0.05$ indicated statistical significance.

\section{Conclusions}

Our result suggested that a daily recommended dose of CCP administered in the absence of training significantly improved grip strength; efficiently relieved fatigue-associated biochemical indices, including lactate and ammonia levels; and ameliorated skeletal muscle injury induced by acute exercise challenge, but had no effect on endurance performance. Therefore, further investigation is required to elucidate the effects of CCP supplementation combined with exercise training on exercise performance and physical fatigue. In conclusion, our study provides experiment-based evidence to 
support traditional claims regarding the antifatigue effect of CCP supplementation and suggests that $\mathrm{CCP}$ can be used as an ergogenic and antifatigue agent.

\section{Acknowledgments}

The corresponding author acknowledges the Ministry of Science and Technology (MOST) of Taiwan, the successor to the National Science Council (grant no. NSC101-2410-H-037-016-MY3), for financial support. The authors thank Melissa Morgan, M.A.T. (Wallace Academic Editing, Taipei City, Taiwan) for her careful reading of the manuscript. The authors thank Mr. Wen-Ching Huang for providing technical assistance in animal experiments and Chien-Chao Chiu for conducting histological examination.

\section{Author Contributions}

Chi-Chang Huang, and Mei-Chich Hsu designed the experiments. Chi-Chang Huang and Hui-Ling Chao carried out the laboratory experiments. Chin-Shan Ho measured forelimb grip strength. Chi-Chang Huang and Yi-Ming Chen analyzed the data, interpreted the results, prepared figures, and wrote the manuscript. Chi-Chang Huang and Mei-Chich Hsu contributed reagents, materials, analysis tools. Chi-Chang Huang, Nai-Wen Kan and Mei-Chich Hsu edited and revised the manuscript. All authors discussed and approved the final manuscript.

\section{Conflict of Interest}

All authors declare no conflicts of interest.

\section{References}

1. Wu, F.; Li, H.; Jin, L.; Li, X.; Ma, Y.; You, J.; Li, S.; Xu, Y. Deer antler base as a traditional Chinese medicine: A review of its traditional uses, chemistry and pharmacology. J. Ethnopharmacol. 2013, 145, 403-415.

2. Liu, Y.; Zhang, G.J.; Sun, S.Q.; Noda, I. Study on similar traditional Chinese medicines cornu Cervi pantotrichum, cornu Cervi and cornu Cervi degelatinatum by FT-IR and 2D-IR correlation spectroscopy. J. Pharm. Biomed. Anal. 2010, 52, 631-635.

3. Zhao, L.; Luo, Y.C.; Wang, C.T.; Ji, B.P. Antioxidant activity of protein hydrolysates from aqueous extract of velvet antler (Cervus elaphus) as influenced by molecular weight and enzymes. Nat. Prod. Commun. 2011, 6, 1683-1688.

4. Tseng, S.H.; Sung, H.C.; Chen, L.G.; Lai, Y.J.; Wang, K.T.; Sung, C.H.; Wang, C.C. Effects of Velvet Antler with Blood on Bone in Ovariectomized Rats. Molecules 2012, 17, 10574-10585.

5. Kim, K.S.; Choi, Y.H.; Kim, K.H.; Lee, Y.C.; Kim, C.H.; Moon, S.H.; Kang, S.G.; Park, Y.G. Protective and anti-arthritic effects of deer antler aqua-acupuncture (DAA), inhibiting dihydroorotate dehydrogenase, on phosphate ions-mediated chondrocyte apoptosis and rat collagen-induced arthritis. Int. Immunopharmacol. 2004, 4, 963-973. 
6. Dragoni, F.; Minotti, C.; Palumbo, G.; Faillace, F.; Redi, R.; Bongarzoni, V.; Avvisati, G. As compared to kaolin clotting time, silica clotting time is a specific and sensitive automated method for detecting lupus anticoagulant. Thromb. Res. 2001, 101, 45-51.

7. Luo, J.; Yan, D.; Zhang, D.; Feng, X.; Yan, Y.; Dong, X.; Xiao, X. Substitutes for endangered medicinal animal horns and shells exposed by antithrombotic and anticoagulation effects. J. Ethnopharmacol. 2011, 136, 210-216.

8. Zhang, L.; Wang, Y.; Wang, L.Z.; Gao, X.M. Immunopotentiating effect of a 'Yang'-promoting formula of traditional Chinese medicine on aged female BALB/c mice. Phytother. Res. 2004, 18, 857-861.

9. Mehta, R.K.; Agnew, M.J. Influence of mental workload on muscle endurance, fatigue, and recovery during intermittent static work. Eur. J. Appl. Physiol. 2012, 112, 2891-2902.

10. Fitts, R.H. Cellular mechanisms of muscle fatigue. Physiol. Rev. 1994, 74, 49-94.

11. Coombes, J.S.; Rowell, B.; Dodd, S.L.; Demirel, H.A.; Naito, H.; Shanely, R.A.; Powers, S.K. Effects of vitamin E deficiency on fatigue and muscle contractile properties. Eur. J. Appl. Physiol. 2002, 87, 272-277.

12. Nybo, L. CNS fatigue and prolonged exercise: Effect of glucose supplementation. Med. Sci. Sports Exerc. 2003, 35, 589-594.

13. You, L.; Zhao, M.; Regenstein, J.M.; Ren, J. In vitro antioxidant activity and in vivo anti-fatigue effect of loach (Misgurnus anguillicaudatus) peptides prepared by papain digestion. Food Chem. 2011, 124, 188-194.

14. Huang, C.C.; Hsu, M.C.; Huang, W.C.; Yang, H.R.; Hou, C.C. Triterpenoid-rich extract from Antrodia camphorata improves physical fatigue and exercise performance in mice. Evid. Based Complement. Alternat. Med. 2012, 2012, 364741.

15. Wang, S.Y.; Huang, W.C.; Liu, C.C.; Wang, M.F.; Ho, C.S.; Huang, W.P.; Hou, C.C.; Chuang, H.L.; Huang, C.C. Pumpkin (Cucurbita moschata) fruit extract improves physical fatigue and exercise performance in mice. Molecules 2012, 17, 11864-11876.

16. Chen, W.C.; Huang, W.C.; Chiu, C.C.; Chang, Y.K.; Huang, C.C. Whey protein improves exercise performance and biochemical profiles in trained mice. Med. Sci. Sports Exerc. 2014, doi:10.1249/MSS.0000000000000272.

17. Syrotuik, D.G.; MacFadyen, K.L.; Harber, V.J.; Bell, G.J. Effect of elk velvet antler supplementation on the hormonal response to acute and chronic exercise in male and female rowers. Int. J. Sport Nutr. Exerc. MeTable 2005, 15, 366-385.

18. Brancaccio, P.; Maffulli, N.; Limongelli, F.M. Creatine kinase monitoring in sport medicine. Br. Med. Bull. 2007, 81-82, 209-230.

19. Cairns, S.P. Lactic acid and exercise performance: Culprit or friend? Sports Med. 2006, 36, 279-291.

20. Carvalho-Peixoto, J.; Alves, R.C.; Cameron, L.C. Glutamine and carbohydrate supplements reduce ammonemia increase during endurance field exercise. Appl. Physiol. Nutr. MeTable 2007, 32, 1186-1190.

21. Suh, S.H.; Paik, I.Y.; Jacobs, K. Regulation of blood glucose homeostasis during prolonged exercise. Mol. Cells 2007, 23, 272-279.

22. Fujii, N.; Jessen, N.; Goodyear, L.J. AMP-activated protein kinase and the regulation of glucose transport. Am. J. Physiol. Endocrinol. MeTable 2006, 291, E867-E877. 
23. Zhang, H.; Wanwimolruk, S.; Coville, P.F.; Schofield, J.C.; Williams, G.; Haines, S.R.; Suttie, J.M. Toxicological evaluation of New Zealand deer velvet powder. Part I: acute and subchronic oral toxicity studies in rats. Food Chem. Toxicol. 2000, 38, 985-990.

24. Wu, R.E.; Huang, W.C.; Liao, C.C.; Chang, Y.K.; Kan, N.W.; Huang, C.C. Resveratrol protects against physical fatigue and improves exercise performance in mice. Molecules 2013, 18, 4689-4702.

Sample Availability: Not available.

(C) 2014 by the authors; licensee MDPI, Basel, Switzerland. This article is an open access article distributed under the terms and conditions of the Creative Commons Attribution license (http://creativecommons.org/licenses/by/3.0/). 